\title{
Cutaneous Head and Neck Squamous Cell Carcinoma with Regional Metastases: The Prognostic Importance of Soft Tissue Metastases and Extranodal Spread
}

\author{
Wendy Kelder, MD, PhD ${ }^{1,2}$, Ardalan Ebrahimi, MBBS (Hons), FRACS ${ }^{1}$, Veronique-Isabelle Forest, MSc, FRCSC ${ }^{1}$, \\ Kan Gao, BEng ${ }^{1}$, Rajmohan Murali, MBBS, MD, FRCPA ${ }^{3}$, and Jonathan R. Clark, MBBS FRACS ${ }^{1}$ \\ ${ }^{1}$ Sydney Head and Neck Cancer Institute, Sydney Cancer Center, Royal Prince Alfred and Liverpool Hospitals, Sydney, \\ Australia; ${ }^{2}$ Department of Surgical Oncology, University Medical Center Groningen, Groningen, The Netherlands; \\ ${ }^{3}$ Human Oncology and Pathogenesis Program, Memorial Sloan-Kettering Cancer Center, New York, NY
}

\begin{abstract}
Background. Extranodal spread (ENS) is an established adverse prognostic factor in metastatic cutaneous squamous cell carcinoma (cSCC); however, the clinical significance of soft tissue metastases (STM) is unknown. The aim of this study was to evaluate the prognosis of patients with STM from head and neck cSCC, and to compare this with that of node metastases with and without ENS.

Methods. Patients with cSCC metastatic to the parotid and/or neck treated by primary surgical resection between 1987 and 2007 were included. Metastatic nodes $>3 \mathrm{~cm}$ in size were an exclusion criterion. A Cox proportional hazard model was used to determine the effect of STM adjusting for other relevant prognostic factors.

Results. The population included 164 patients with a median follow-up of 26 months. There were 8 distant and 37 regional recurrences. There were 22 were cancer-specific deaths, and 29 patients died. STM was a significant predictor of reduced overall (hazard ratio 3.3 ; 95\% confidence interval $1.6-6.4 ; P=0.001)$ and disease-free survival (hazard ratio $2.4 ; 95 \%$ confidence interval 1.4-4.1; $P=0.001)$ when compared to patients with node disease with or without ENS. After adjusting for covariates, STM and number of involved nodes were significant independent predictors of overall and disease-free survival.
\end{abstract}

(C) The Author(s) 2011. This article is published with open access at Springerlink.com

First Received: 26 December 2010;

Published Online: 9 August 2011

J. R. Clark, MBBS FRACS

e-mail: jcjc@tpg.com.au
Conclusions. In metastatic cSCC of the head and neck, the presence of STM is an independent predictor of reduced survival and is associated with a greater adverse effect than ENS alone.

In countries with large white populations and high ambient solar exposure such as Australia and New Zealand, cutaneous squamous cell carcinoma (cSCC) is a major public health problem. ${ }^{1}$ Most cases of cSCC arise in the head and neck, and regional metastases to parotid and/or cervical lymph nodes are thought to occur in approximately $5 \%$ of patients, decreasing 5-year survival rates to $46-70 \% .^{2-9}$

Although extension of metastatic squamous cell carcinoma (SCC) beyond the lymph node capsule (extracapsular spread or extranodal spread, ENS) is a well-established adverse prognostic factor, there is no published evidence regarding the clinical significance of soft tissue metastases (STM) in cSCC. ${ }^{6,10}$ STM are defined as free soft tissue tumor deposits lacking continuity with the primary tumor and without discernible associated lymph node tissue. It remains unclear whether these are true extranodal metastases or whether they represent lymph nodes replaced by tumor to such an extent that the underlying node is unrecognizable. ${ }^{11}$ In the latter case, STM may represent an advanced stage of node disease beyond ENS, and if this is the case, larger tumor size and reduced locoregional control would be expected. This notion is supported by the known correlation between the size of involved lymph nodes and the presence of ENS. ${ }^{12}$ Several studies of mucosal head and neck SCC have demonstrated an association between STM and unfavorable outcomes, comparable to the presence of ENS. ${ }^{11-16}$ 
It was unclear from our clinical experience whether STM were similar to node deposits with ENS or whether they represented a distinct entity with more aggressive tumor biology. The goal of the current study was to evaluate the prognostic significance of STM in head and neck $\mathrm{cSCC}$, and to compare it with that of node metastases with and without ENS.

\section{MATERIALS AND METHODS}

Clinicopathologic data from patients treated at the Sydney Head and Neck Cancer Institute, Royal Prince Alfred Hospital, Sydney, Australia, were recorded prospectively on a computerized database since 1987 . The database contains information on patient demographics, clinical and pathologic staging, treatment details, histopathologic features, duration of follow-up, and status at last visit. All patients with cSCC metastatic to the parotid and/or neck treated by primary surgical resection with curative intent between 1987 and 2007 were identified. The pathology details recorded in the database were cross-checked with the original pathology reports and the database was updated where necessary. Slides of tumors from all selected patients were reexamined by a pathologist experienced in the examination of head and neck tumors (R.M.). ENS was defined as extension of lymph node metastases of SCC beyond the nodal capsule, and STMs were defined as free soft tissue tumor deposits lacking continuity with the primary tumor and without discernible associated lymph node tissue. The presence, size, and number of STM were measured and added to the existing database. To exclude the effect of bulky tumors, only patients with metastatic nodes $\leq 3 \mathrm{~cm}$ in size were included.

Data were collated and filtered by Microsoft Excel, and statistical analysis was performed by SPSS version 16.0 (SPSS, Chicago, IL). Categorical data were analyzed by chi-square test or Fisher's exact test, normally distributed continuous data by $t$-test, and nonparametric data by Kruskal-Wallis test where appropriate. All statistics were two sided, and a $P$ value of $<0.05$ was considered statistically significant. Overall survival was calculated from the date of surgery to date of death or last follow-up. Diseasefree survival was calculated from the date of surgery to date of last follow-up including date of death from any cause if there were no recurrence or date of first disease recurrence. Differences in survival were determined by univariate Cox regression analysis, and survival curves were generated by the Kaplan-Meier method. A Cox proportional hazard model was used to adjust for the effect of other statistically significant covariates and potential confounders, including postoperative radiotherapy and the number of tumor deposits, by means of purposeful selection of covariates to develop a final multivariable model according to the method of Hosmer et al. ${ }^{17}$

\section{RESULTS}

\section{Study Population}

The study population included 164 patients, 142 men $(86.6 \%)$ and 22 women (13.4\%), with a median age at surgery of 73 years (range 25-98 years) and a median follow-up period of 26 months. Surgical treatment consisted of parotidectomy with neck dissection in 129 patients $(78.7 \%)$, neck dissection alone in $14(8.5 \%)$, and parotidectomy alone in 21 (12.8\%). Adjuvant radiotherapy, at a median dose of $54 \mathrm{~Gy}$ (range 32-66 Gy), was administered to 131 patients $(79.9 \%)$.

\section{Pathology of Metastatic Disease}

The median number of either positive nodes or metastatic deposits was 1 , while the mean number was 3.7 (range 1-68). Most patients $(104,63.4 \%)$ had only one affected node or STM. The margins of excision were clear in 83 patients $(50.6 \%)$, involved in 27 patients $(16.5 \%)$, and close $(<5 \mathrm{~mm})$ in 38 patients $(23.2 \%)$, and there was no information on margin status in 16 patients $(9.8 \%)$. Fifty-eight patients $(35.4 \%)$ had node metastases without ENS or STM, 51 (31.1\%) had ENS and STM, 33 (20.1\%) had ENS alone, and 22 (13.4\%) had STM alone (Fig. 1). The median size of the largest node or STM was $1.8 \mathrm{~cm}$. The mean diameter of involved nodes (in the selected group of patients with tumors $\leq 3 \mathrm{~cm}$ ) without ENS, nodes with ENS, and STMs was $1.9 \mathrm{~cm}$ (range $0.5-3.0 \mathrm{~cm}$ ), $1.7 \mathrm{~cm}$ (range $0.6-2.5 \mathrm{~cm}$ ), and $2.1 \mathrm{~cm}$ (range 1.0-3.0 $\mathrm{cm})$, respectively $(P=0.085$; Kruskal-Wallis test). Seventy-one patients $(43 \%)$ had nodes $<1.5 \mathrm{~cm}$.

During the follow up there were eight distant and 37 regional recurrences. Twenty-nine patients died; there were

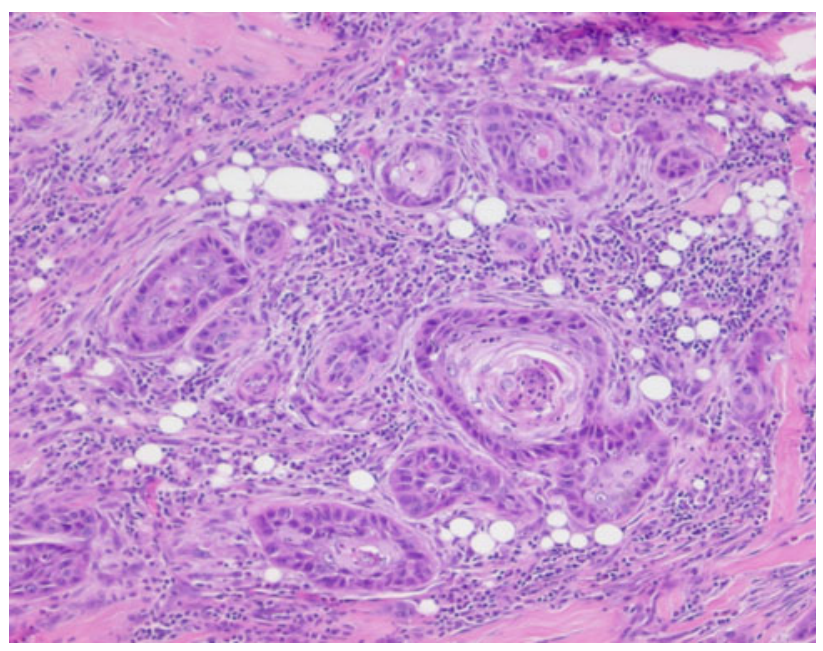

FIG. 1 STM of cutaneous SCC 
FIG. 2 Kaplan-Meier curves according to the presence or absence of STM for (a) overall survival and (b) disease-free survival

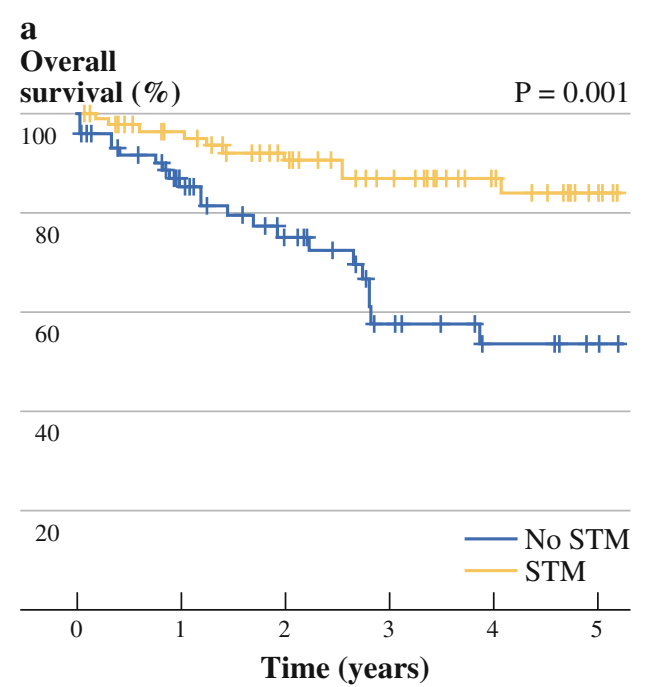

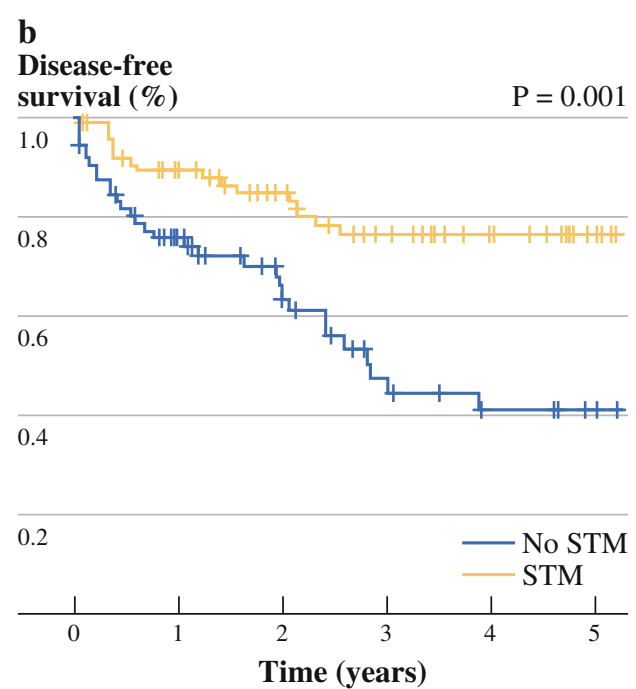

22 cancer-specific deaths. STM was a significant predictor of reduced overall [hazard ratio (HR) 3.3; 95\% confidence interval (CI) 1.6-6.4; $P=0.001]$ and disease-free survival (HR, 2.4; 95\% CI $1.4-4.1 ; P=0.001$ ) when compared to patients with node disease with or without ENS (Fig. 2). The presence of ENS was also a significant predictor of survival when compared to patients without ENS or soft tissue deposits. Other significant predictors of survival on univariate analysis are summarized in Table 1. After adjusting for other covariates, STM and number of involved nodes (or tumor deposits) were significant independent predictors of overall and disease-free survival, but ENS was not (Table 2).

To compare the prognostic impact of STM and ENS, the study population was divided into four groups: STM and ENS; STM alone; ENS alone; and node disease without ENS or STM. The adjusted Cox regression curves in Fig. 3 show that the worst outcomes were associated with STM with or without associated ENS, while node disease without STM or ENS was associated with the most favorable outcomes, and ENS alone had an intermediate prognosis.

When the subset of patients with STM alone $(n=22)$ were compared with those with ENS alone $(n=33)$, patients with STM alone had significantly reduced overall survival (HR 4.1; 95\% CI 1.0-16.8; $P=0.048$ ) when compared to those with ENS alone after adjusting for the effect of age and number of lymph nodes (Fig. 4).

\section{DISCUSSION}

The results of the present study suggest that the presence of STMs is associated with a worse prognosis than the presence of node metastases (with or without ENS). We were unable to confirm that ENS in node metastases $\leq 3 \mathrm{~cm}$ in size is associated with reduced survival after adjusting
TABLE 1 Univariate survival analysis

\begin{tabular}{|c|c|c|c|}
\hline Variable & HR & $95 \% \mathrm{CI}$ & $P$ value \\
\hline \multicolumn{4}{|l|}{ Overall survival } \\
\hline Age (continuous) & 1.04 & $1.00-1.08$ & 0.031 \\
\hline Involved margin & 1.62 & $0.73-3.56$ & 0.233 \\
\hline \multicolumn{4}{|l|}{ Maximal lesion size } \\
\hline Size (continuous) & 1.02 & $0.96-1.09$ & 0.484 \\
\hline$>1.5$ versus $\leq 1.5 \mathrm{~cm}$ & 1.28 & $0.56-2.92$ & 0.565 \\
\hline \multicolumn{4}{|l|}{ No. of nodes } \\
\hline $2-3$ nodes versus 1 node & 1.03 & $0.38-2.83$ & 0.949 \\
\hline$\geq 4$ nodes versus 1 node & 5.13 & $2.35-11.20$ & $<0.001$ \\
\hline STM & 3.25 & $1.63-6.49$ & 0.001 \\
\hline $\mathrm{ENS}^{\mathrm{a}}$ & 2.59 & $1.30-5.16$ & 0.007 \\
\hline Adjuvant radiotherapy ${ }^{\mathrm{a}}$ & 0.72 & $0.33-1.57$ & 0.405 \\
\hline \multicolumn{4}{|l|}{ Disease-free survival } \\
\hline Age & 1.02 & $0.99-1.04$ & 0.220 \\
\hline Involved margin & 2.18 & $1.20-3.96$ & 0.010 \\
\hline \multicolumn{4}{|l|}{ Maximal lesion size } \\
\hline Size (continuous) & 1.02 & $0.98-1.07$ & 0.390 \\
\hline$>1.5$ versus $\leq 1.5 \mathrm{~cm}$ & 1.40 & $0.73-2.67$ & 0.310 \\
\hline \multicolumn{4}{|l|}{ No. of nodes } \\
\hline $2-3$ nodes versus 1 node & 1.73 & $0.84-3.57$ & 0.135 \\
\hline$\geq 4$ nodes versus 1 node & 4.94 & $2.51-9.73$ & $<0.001$ \\
\hline $\mathrm{STM}^{\mathrm{a}}$ & 2.42 & $1.42-4.14$ & 0.001 \\
\hline $\mathrm{ENS}^{\mathrm{a}}$ & 2.01 & $1.17-3.45$ & 0.012 \\
\hline Adjuvant radiotherapy & 0.79 & $0.42-1.50$ & 0.471 \\
\hline
\end{tabular}

${ }^{\text {a }}$ Different reference categories for STM and ENS mean hazards are not comparable

for the effect of STM and number of nodes involved. However, it is likely that the study is insufficiently powered to detect a statistically significant difference because the adverse effect of ENS was considerably less than that of 
TABLE 2 Final multivariable survival analysis models

\begin{tabular}{lccr}
\hline Variable & HR & $95 \%$ CI & $P$ value \\
\hline Overall survival & & & \\
Age (continuous) & 1.03 & $1.00-1.07$ & 0.072 \\
No. of nodes & 2.34 & $1.49-3.66$ & $<0.001$ \\
STM & 2.91 & $1.44-5.88$ & 0.003 \\
Adjuvant radiotherapy & 0.63 & $0.28-1.40$ & 0.256 \\
ENS & 1.54 & $0.72-3.29$ & 0.263 \\
Disease-free survival & & & \\
Age & 1.01 & $0.98-1.04$ & 0.487 \\
Margin & 0.96 & $0.49-1.86$ & 0.899 \\
No. of nodes & 2.31 & $1.61-3.32$ & $<0.001$ \\
STM & 2.35 & $1.35-4.07$ & 0.002 \\
Adjuvant radiotherapy & 0.75 & $0.39-1.44$ & 0.385 \\
ENS & 1.30 & $(0.71-2.38)$ & 0.395 \\
\hline
\end{tabular}

STM (type II error). This lends support to the argument that STMs, rather than representing lymph nodes completely replaced by tumor, may instead represent a distinct biologic entity (namely deposits of tumor in soft tissue, not associated with lymph nodes).

Because number and size of tumor deposits are important prognostic factors and size of the tumor is related to the presence of ENS and STM, we tried to exclude the effect of bulky tumors by only selecting patients with tumors up to $3 \mathrm{~cm}$ as supported by the N1S3 and current tumor, node, metastasis staging systems. ${ }^{10,12,18}$ In this subgroup, the presence of STM was a statistically significant adverse prognostic factor, and the effect was even more pronounced in patients with both STM and ENS.

A limitation of this study is that primary tumor information is not evaluated. Other factors that could confound the effect of STM include perineural invasion, lymphovascular invasion, depth of invasion, tumor grade, and immune status of the patient. ${ }^{3,7}$ Many of these factors were adopted in the 7th edition of the American Joint Committee on Cancer/International Union Against Cancer staging system for cSCC. The prognostic significance of primary tumor factors in patients with node metastases is unknown. Patients in this study were only referred with node metastases, primary tumor data was often unavailable and many patients have either no identifiable primary or multiple potential primaries.

Extranodal spread and soft tissue metastases are common findings in patients with metastatic cutaneous and mucosal SCC, one or the other being present in most patients. ${ }^{6},{ }^{11-16}$ Generally, the distinction has been ignored by pathologists and clinicians and both have been grouped together. Studies in mucosal SCC have shown that the presence of both STM and/or ENS is related to reduced survival, but it is not clear from those studies whether STM and ENS are identical. ${ }^{11-16}$ Partly this is because the term "soft tissue deposit or metastasis" is used rather imprecisely and may include direct extension of tumor cells from the primary site or even ENS of tumor from the lymph node into the soft tissues of the neck. In an attempt to be more specific we defined STM as "free soft tissue tumor deposits lacking continuity with the primary tumor and without discernible associated lymph node tissue." In melanoma, "microscopic satellitosis" represents a parallel adverse prognostic factor that has been more extensively studied and strictly defined as "any discontinuous nest of metastatic cells more than $0.05 \mathrm{~mm}$ in diameter that are clearly separated by normal dermis from the main invasive component of melanoma by a distance of at least $0.3 \mathrm{~mm} .{ }^{19}$ Despite the similarity, we believe that these are different biologic entities as STM mainly occur in node basins rather than as dermal deposits within close proximity to the primary tumor. Regardless, a strict definition such as that adopted for microscopic satellitosis would enable reliable and reproducible estimates of STM's prognostic significance in future studies. ${ }^{20-22}$

STM could occur via spread of tumor cells through lymphatic channels draining the primary tumor or through perineural or vascular routes. Tumor cells that spread through lymphatics eventually reach lymph nodes, where they will either be contained by the local immune system or will overcome the local host defense and may eventually replace the whole lymph node with tumor. It has been demonstrated in animal models that some tumor cells escape the lymphatics or travel through small vessels and end up as free tumor deposits in the soft tissues. ${ }^{23}$ Tumors escaping lymphatic channels may possess cellular properties that promote growth and dissemination and may avoid some of the immune regulation that normally occurs in lymph nodes, whereas tumors with direct vascular invasion may be more prone to distant spread. The real explanation is likely to be complicated and multifactorial, and merits further study.

In this series, the predominant site of recurrence was within the parotid and neck suggesting that the focus of treatment should remain on optimizing locoregional control. There is good evidence that combined modality treatment with surgery and adjuvant radiotherapy improves locoregional control and survival in head and neck cSCC. $^{5,24-26}$ However, radiotherapy is associated with marked acute and long-term toxicity, and a recent multicenter study involving our institution suggests there is a low-risk group of patients with N1S3 stage I disease (single lymph node $\leq 3 \mathrm{~cm}$ ) and no ENS or STM that may be suitable for treatment with surgery alone. ${ }^{10,27,28}$ Conversely, the results of the Trans-Tasman Radiation Oncology Group trial (Postoperative Skin Trial 05.01; http://www.trog.com.au/) are awaited to determine whether 
FIG. 3 Cox proportional hazard regression comparing

(a) overall survival and

(b) disease-free survival, according to presence of ENS and STM versus STM alone versus ENS alone versus node disease without ENS or STM; adjusted by age, number of involved nodes and adjuvant radiotherapy

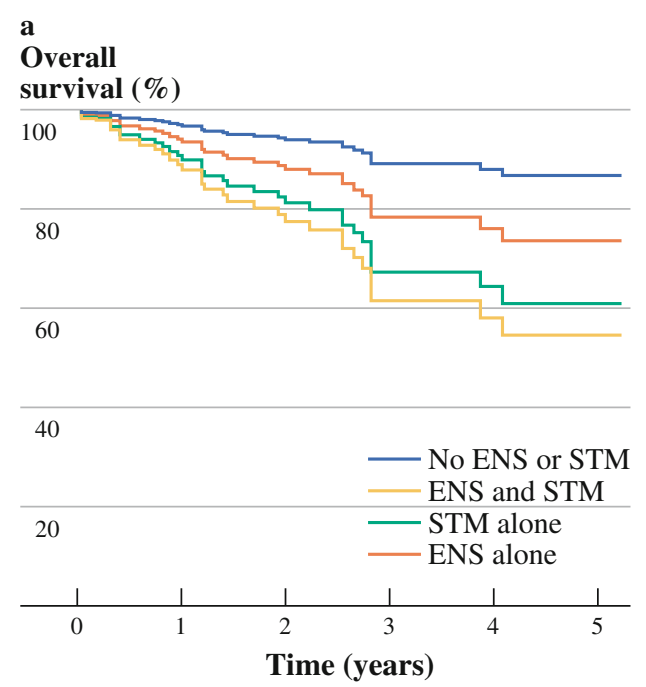

FIG. 4 Cox proportional hazard regression comparing (a) overall survival and (b) disease-free survival, according to presence of STM alone versus ENS alone; adjusted by age and number of involved nodes

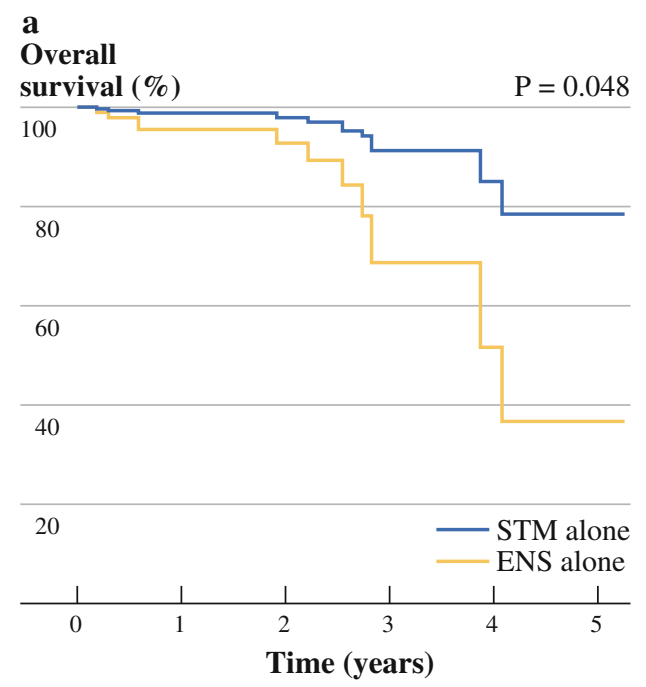

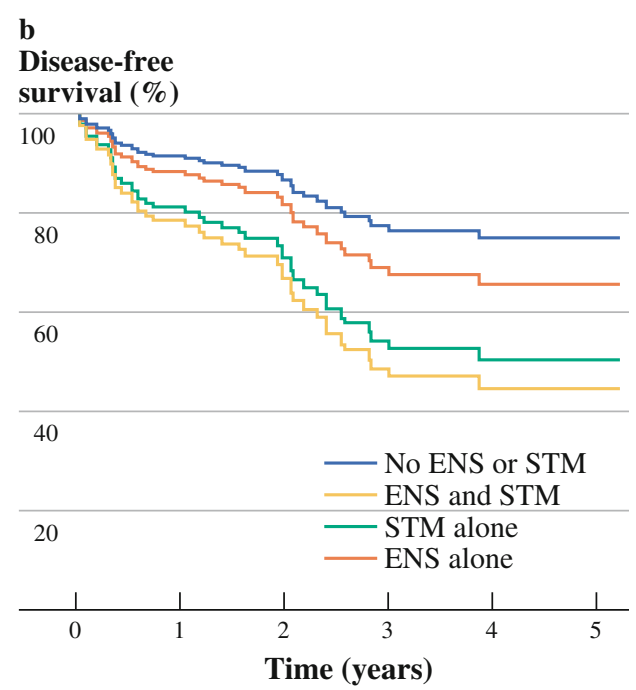

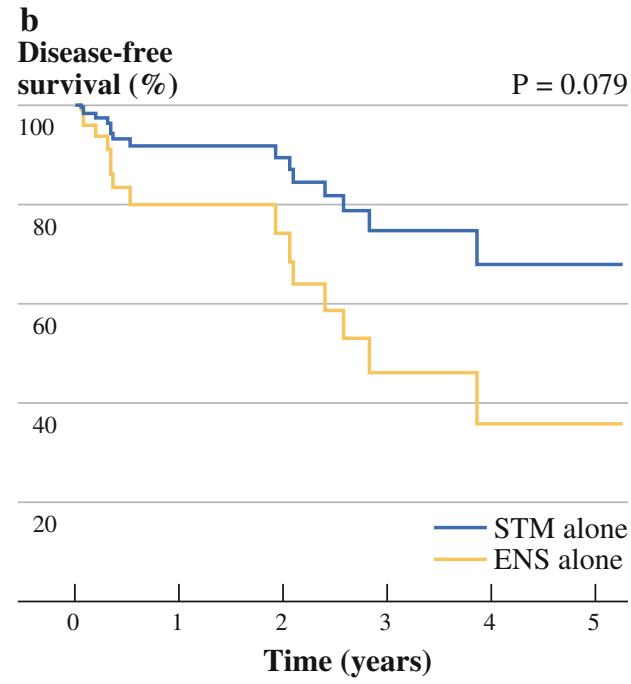

intensification of treatment regimens with adjuvant chemoradiation may be beneficial for high-risk metastatic cSCC of the head and neck. Hence, accurate prognostic information remains critical to making decisions regarding deescalation or intensification of adjuvant therapy.

Soft tissue metastases have a comparable adverse effect on survival to multiple lymph node metastases. Therefore, adjuvant radiotherapy should be administered to all patients with STM, even if the metastasis is a small single deposit. We are cautious about making recommendations for patients with ENS, despite being unable to demonstrate a marked effect. Therefore, conventional multimodal therapy should be considered standard on the basis of evidence from multiple studies which have shown ENS to be an adverse prognostic factor, even though they are likely to have grouped ENS and STM together. 6,7,10

The pathologic examination and reporting of parotid and neck dissection specimens is paramount to obtaining reliable prognostic information on which to base treatment decisions. It is essential that examination of all tumor deposits and lymph nodes (including the smallest nodes) is performed for the presence of ENS and STM, along with measurement of the size and number of metastases. ${ }^{10}$ This approach has also been recommended in other solid tumors, for example colorectal cancers, in which the majority of metastases are found in lymph nodes that are smaller than $5 \mathrm{~mm}$ in size, and even small metastatic tumor deposits have a negative prognostic impact. ${ }^{29-31}$ As in many solid tumors, a low number of examined nodes probably represents understaging, and is associated with poorer prognosis. ${ }^{31}$ Clearly, adequate staging requires not only a meticulous surgical technique but also a detailed pathologic evaluation.

In conclusion, in metastatic cSCC of the head and neck, the presence of STM (with or without associated ENS) is a statistically significant independent predictor of reduced 
survival and is associated with more unfavorable outcomes than the presence of ENS alone. Further work is required to determine the biologic mechanisms underlying this finding. Similar to the guidelines in melanoma, pathology reports of head and neck cSCC should include the number and size of affected nodes, the presence of ENS, as well as the presence, number, and size of STMs. The threshold for aggressive multimodal treatment should be low in the presence of STM.

ACKNOWLEDGMENT W.K. was supported as a clinical and research fellow by the Dutch Cancer Society (grant RUG 2008-4382).

OPEN ACCESS This article is distributed under the terms of the Creative Commons Attribution Noncommercial License which permits any noncommercial use, distribution, and reproduction in any medium, provided the original author(s) and source are credited.

\section{REFERENCES}

1. Staples M, Marks R, Giles G. Trends in the incidence of nonmelanocytic skin cancer (NMSC) treated in Australia, 19851995: are primary prevention programs starting to have an effect? Int J Cancer. 1998;78:144-8.

2. Alam M, Ratner D. Cutaneous squamous-cell carcinoma. $N$ Engl J Med. 2001;344:975-983.

3. Brantsch KD, Meisner C, Schonfisch B, et al. Analysis of risk factors determining prognosis of cutaneous squamous-cell carcinoma: a prospective study. Lancet Oncol. 2008;9:713-20.

4. Czarnecki D, Staples M, Mar A, Giles G, Meehan C. Metastases from squamous cell carcinoma of the skin in southern Australia. Dermatology. 1994;189:52-4.

5. Jol JA, van Velthuysen ML, Hilgers FJ, Keus RB, Neering H, Balm AJ. Treatment results of regional metastasis from cutaneous head and neck squamous cell carcinoma. Eur J Surg Oncol. 2003;29:81-6.

6. Oddone N, Morgan GJ, Palme CE, et al. Metastatic cutaneous squamous cell carcinoma of the head and neck: the Immunosuppression, Treatment, Extranodal Spread, and Margin Status (ITEM) prognostic score to predict outcome and the need to improve survival. Cancer. 2009;115:1883-91.

7. Veness MJ, Palme CE, Morgan GJ. High-risk cutaneous squamous cell carcinoma of the head and neck: results from 266 treated patients with metastatic lymph node disease. Cancer. 2006;106:2389-96.

8. Taylor BW, Brant TA, Mendenhall NP, et al. Carcinoma of the skin metastatic to parotid area lymph nodes. Head Neck. 1991; 13:427-33.

9. O'Brien CJ, McNeil EB, McMahon JD, Pathak I, Lauer CS. Incidence of cervical node involvement in metastatic cutaneous malignancy involving the parotid gland. Head Neck. 2001;23:744-8.

10. Forest VI, Clark JJ, Veness MJ, Milross C. N1S3: a revised staging system for head and neck cutaneous squamous cell carcinoma with lymph node metastases: results of 2 Australian cancer centers. Cancer. 2010;116:1298-304.

11. Jose J, Moor JW, Coatesworth AP, Johnston C, MacLennan K. Soft tissue deposits in neck dissections of patients with head and neck squamous cell carcinoma: prospective analysis of prevalence, survival, and its implications. Arch Otolaryngol Head Neck Surg. 2004;130:157-60.

12. Puri SK, Fan CY, Hanna E. Significance of extracapsular lymph node metastases in patients with head and neck squamous cell carcinoma. Curr Opin Otolaryngol Head Neck Surg. 2003; 11:119-23.

13. MacLennan K, Jose J, Ferlito A, et al. Cervical soft tissue metastases in head and neck cancer. Acta Otolaryngol. 2003; 123:336-9.

14. Coatesworth AP, MacLennan K. Squamous cell carcinoma of the upper aerodigestive tract: the prevalence of microscopic extracapsular spread and soft tissue deposits in the clinically N0 neck. Head Neck. 2002;24:258-61.

15. Violaris NS, O’Neil D, Helliwell TR, Caslin AW, Roland NJ, Jones AS. Soft tissue cervical metastases of squamous carcinoma of the head and neck. Clin Otolaryngol. 1994;19:394-9.

16. Shah JP, Cendon RA, Farr HW, Strong EW. Carcinoma of the oral cavity. Factors affecting treatment failure at the primary site and neck. Am J Surg. 1976;132:504-7.

17. Hosmer D, Lemeshow S, May S. Applied survival analysis: regression modeling of time-to-event data. 2nd ed. Hoboken: Wiley; 2008

18. American Joint Committee on Cancer. Cancer staging handbook: TNM classification of malignant tumors. New York: Springer; 2002.

19. Balch CM, Gershenwald JE, Soong S, et al. Final version of 2009 AJCC melanoma staging and classification. J Clin Oncol. 2009; 27:6199-206.

20. Kimsey TF, Cohen T, Patel A, Busam KJ, Brady MS. Microscopic satellitosis in patients with primary cutaneous melanoma: implications for nodal basin staging. Ann Surg Oncol. 2009;16:1176-83.

21. Balch CM. Microscopic satellites around a primary melanoma: another piece of the puzzle in melanoma staging. Ann Surg Oncol. 2009;16:1092-4.

22. Rao UN, Ibrahim J, Flaherty LE, Richards J, Kirkwood JM. Implications of microscopic satellites of the primary and extracapsular lymph node spread in patients with high-risk melanoma: pathologic corollary of Eastern Cooperative Oncology Group Trial E1690. J Clin Oncol. 2002;20:2053-7.

23. Cabanillas R, Secades P, Rodrigo JP, Astudillo A, Suárez C, Chiara MD. Orthotopic murine model of head and neck squamous cell carcinoma. Acta Otorrinolaringol Esp. 2005;56:89-95.

24. Shimm DS, Wilder RB. Radiation therapy for squamous cell carcinoma of the skin. Am J Clin Oncol. 1991;14:383-6.

25. Veness MJ, Palme CE, Smith M, Cakir B, Morgan GJ, Kalnins I. Cutaneous head and neck squamous cell carcinoma metastatic to cervical lymph nodes (nonparotid): a better outcome with surgery and adjuvant radiotherapy. Laryngoscope. 2003;113:1827-33.

26. Veness MJ, Morgan GJ, Palme CE, Gebski V. Surgery and adjuvant radiotherapy in patients with cutaneous head and neck squamous cell carcinoma metastatic to lymph nodes: combined treatment should be considered best practice. Laryngoscope. 2005; $115: 870-5$.

27. August M, Wang J, Plante D, Wang CC. Complications associated with therapeutic neck radiation. J Oral Maxillofac Surg. 1996;54:1409-15.

28. Ebrahimi A, Clark JR, Lorincz BB, Milross CG, Veness MJ. Metastatic head and neck cutaneous squamous cell carcinoma: defining a low-risk patient. Head Neck. 2011. doi:10.1002/hed.21743.

29. Haboubi NY, Abdalla SA, Amini S, et al. The novel combination of fat clearance and immunohistochemistry improves prediction of the outcome of patients with colorectal carcinomas: a preliminary study. Int J Colorectal Dis. 1998;13:99-102.

30. Liefers GJ, Cleton-Jansen AM, van de Velde CJ, et al. Micrometastases and survival in stage II colorectal cancer. $N$ Engl J Med. 1998;339:223-8.

31. Jestin P, Pahlman L, Glimelius B, Gunnarsson U. Cancer staging and survival in colon cancer is dependent on the quality of the pathologists' specimen examination. Eur J Cancer. 2005;41: 2071-8. 